\title{
Veränderung der Bodenunruhe 2020 in Wien durch Covid- 19-Maßnahmen
}

\section{Analyse von Bodenunruhe und Schwingungsquellen}

\author{
Dr. Maria-Theresia Apoloner, Zentralanstalt für Meteorologie und \\ Geodynamik, Wien; \\ DI Helmut Hausmann, Dr. Yan Jia, Dr. Wolfgang Lenhardt
}

\section{Kurzfassung}

Die Maßnahmen, die zur Eindämmung von Corona gesetzt wurden, spiegeln sich auch in der Bodenunruhe in ganz Österreich wieder. Die seismologischen Messstationen des Erdbebendienstes der ZAMG zeigen: der Boden vibriert deutlich weniger, wie sonst nur an Wochenenden, Feiertragen und Ferienzeiten. So können u.a. kleinere Erdbeben detektiert werden, welche eine wichtige Grundlage für Bestimmung der Erdbebengefährdung und somit erdbebengerechtes Bauen sind.

Nicht nur Erdbeben können so besser detektiert werden, die Messdaten aus diesem Zeitraum bieten eine besonders gute Möglichkeit sich mit anthropogenen Quellen auseinander zu setzen: sie können leichter identifiziert und in ihrer Stärke quantifiziert werden. Dazu analysieren wir die Daten der Messstationen der ZAMG in und nahe Wiens mit der Open-Source Software „SeismoRMS“. Anschließend präsentieren wir die Ergebnisse für den Frequenzbereich von $4-14 \mathrm{~Hz}$ mit in der Seismologie standardmäßig verwendeten Darstellungen für Bodenunruhe. Diese Darstellungen ermöglichen bei der Analyse von Bodenschwingungen wichtige Erkenntnisse über zeitliche Muster von unterschiedlichen Quellen wie Verkehr, Industrie, sowie Wetterereignissen. Abschließend präsentieren wir den Ablauf der Signalanalyse eines davor unidentifizierten Messsignals, welches in Wien im Juli 2020 auf allen Stationen messbar war - und natürlichen Ursprungs war.

\footnotetext{
Abstract

Due to the spread of Covid-19, lockdown measures were taken in Austria,affecting not only the population but also the seismic noise across of Austria. Recordings from the seismic sensors of the Seismological Service of Austria showed that the earth vibrates significantly less, comparable to the noise level on weekends or holidays. Therefore, smaller earthquakes could be detected, which supplement earthquake hazard estimations in Austria and earthquake adequate building codes as well.
}

Vortrag Reduzierte Bodenunruhe 2020 durch Corona (Apoloner)

2. Wiener Dynamik Tage 2021 
However, not only earthquakes can possibly be detected better, but also this period of time offers an opportunity to look into anthropogenice noise sources in greater detail, i.e. can be identified and evaluated better than before and after this period. For this reason we analyse continuous data recorded by seismic stations in and around Vienna operated by ZAMG with the open-source "SeismoRMS" package and present typical graphical representations of noise to further the understanding of noise sources. We also show how the different representations facilitate the analysis of noise sources. Finally we present a concept of how to evaluate an unknown non-earthquake signal visible on all stations in Vienna from July 2020 of natural origin.

Vortrag Reduzierte Bodenunruhe 2020 durch Corona (Apoloner) 


\section{Einleitung}

Die klassische Seismologie beschäftigt sich mit der Analyse von Erdbebensignalen. Im städtischen Bereich wird damit unter anderem die Erdbebengefährdung untersucht [1] oder auch Infrastruktur überwacht. Die kontinuierliche Aufzeichnung dient auch der Prüfung ob ÖNORM-Werte überschritten wurden, sowie der Beurteilung der Erdbebensicherheit und Unterstützung des Zivil- und Katastrophenschutzes bei Erdbeben. Dafür müssen nicht nur Schadensbeben aufgezeichnet, sondern auch kleine Beben detektiert werden. Sie treten wesentlich häufiger auf und werden als Grundlage für die Häufigkeitsverteilung und somit die langfristige Erdbebengefährdung benötigt.

Ständige menschliche Aktivität im urbanen Bereich führt zu einer dauerhaft verstärkten Bodenunruhe, welche kleine lokale Erdbebensignale überdeckt, und deren Analyse erschwert. Die Maßnahmen, die zu Beginn 2020 zur Eindämmung von Covid-19 gesetzt wurden, spiegeln sich in der Bodenunruhe weltweit [2] wieder. Die österreichischen Stationen (VIE in Wien und DAVA in Damüls) des Erdbebendienstes der ZAMG [3], die in dieser Studie verwendet wurden zeigten, dass dies auch für Österreich der Fall war.

In Zeiten reduzierter Bodenunruhe können nicht nur tektonische Quellen wie Erdbeben besser detektiert werden, die Messdaten aus diesem Zeitraum bieten eine Chance anthropogene Quellen und andere nicht tektonische Quellen wie etwa Wetterereignisse [4] zu identifizieren. In internationalen Studien wurden bereits verschiedenste Quellen wie etwa Fußballtore [5], ein Marathon [5] oder auch Kirchenglocken [6] identifiziert.

Zunächst analysieren wir in dieser Studie die Veränderung der Bodenunruhe in Wien von Januar bis Juni 2020, welche den Zeitraum der intensivsten Covid-19-Maßnahmen enthält. Wir zeigen unterschiedliche Darstellungsformen und vergleichen die Ergebnisse anschließend mit den Mobilitätsdaten von Google. Zusätzlich analysieren wir Signale welche im Juli 2020 in Wien gemessen und durch ein Wetterereignis verursacht wurden.

\section{Daten}

\subsection{Seismologisches Messnetzwerk in Wien}

Wien eignet sich für eine detaillierte Studie, da das österreichweite Stationsnetzwerk der ZAMG hier durch zwei weitere lokale Netzwerke verdichtet wurde, wie in die Karte in Abbildung 2-1 zeigt. Für die kontinuierliche Überwachung und besonderes die Messung von lokalen Starkbeben wurde mit der MA29 der Stadt Wien ein Netzwerk aus sechs Vortrag Reduzierte Bodenunruhe 2020 durch Corona (Apoloner) 
Starkbebenstationen [7] (Beschleunigungsmessgeräte UMWA, KMWA, SNWA, BGWA, WIWA, ZAWA) installiert. Diese reagieren zwar kaum auf schwache Erdbeben, sind aber bei der Messung von lokalen Starkbeben wichtig, da sie nicht wie Seismometer (Geschwindigkeitsmesser) übersteuern. Das Messnetz wurde 1992 in Betrieb genommen und wurde im Zuge einer Erneuerung der Instrumentierung von einer getriggerten Datenübertragung ab 2017 auf eine kontinuierliche Datenübertragung umgestellt.

Im Rahmen des FFG Projektes GeoTief 3D Explore [8] wurden im Jahr 2019 vier zusätzliche Seismometer (Geschwindigkeitsmessgeräte GNLA, GPRA, GFSA, GAKA) in Wiens Osten aufgebaut. Sie dienen dem Monitoring von kleinen lokalen Erdbeben für die Erdbebengefährdungsabschätzung im Rahmen des Projektes.

\subsection{Vergleichsdaten}

Zum Vergleich zeigen wir Daten der Messstation CONA im Conrad Observatorium (Niederösterreich), deren Lage auf einem Berg in einem Stollen so gewählt wurde, dass sie von menschlicher Aktivität kaum gestört wird.

Weiters sind hier für Wien auch Daten aus anderen Quellen verfügbar, wie etwa die Mobilitätsdaten von Google [9]. Für Wien stehen diese ab dem 15. Februar 2020 zu Verfügung. Sie spiegeln das Mobilitätsverhalten in unterschiedlichen Kategorien (Parks, Wohnstätten, Arbeitsorte, ...) anhand von aggregierten GPS-Daten aus Mobilgeräten wieder.

\section{Methode}

In dieser Studie ermitteln wir die Bodenunruhe als Median der Amplitude der Bodenverschiebung mit der Methodik und den Parametern welche in [2] vorgestellt wurden. Dies hat den Vorteil das starke Ausreißer wie etwa Erdbeben oder kurze transiente Signale z. B. von Gewittern reduziert werden. Aus den Messdaten der vertikalen Komponente wird die spektrale Leistungsdichte (Power Spectral Density, PSD [10]) unter Verwendung der "ObsPy“-Implementierung [11] über 30 Minuten aggregiert. Normalerweise wird diese Darstellung verwendet um langfristig die Qualität eines Messstandortes abzuschätzen. In dieser Art von Darstellung (siehe Abbildung 4-2) wird fast immer das weltweite Referenzmodell [12] gezeigt: die grauen Linien zeigen im globalen Vergleich sehr stark und sehr wenig gestörte Stationen, wobei die Messwerte durch den Standort (Insel, Sedimente, ...), Bauform (Oberfläche, Stollen, Bohrloch, Isolierung,...) als auch Messgerätetyp

Vortrag Reduzierte Bodenunruhe 2020 durch Corona (Apoloner)

2. Wiener Dynamik Tage 2021 
beeinflusst werden können. Klar zu erkennen ist hier das Maximum bei einer Periode von rund 5 Sekunden, welches durch die Brandung von Ozeanen verursacht wird.

Die mittlere spektrale Leistungsdichte wird dann weiter in Amplitude der Bodenunruhe umgerechnet. Dafür wird im Frequenzbereich zwischen 4 und $14 \mathrm{~Hz}$ gemittelt, da in diesem der Einfluss von anthropogenen Quellen besonders stark ist.

Wir berechnen die Bodenunruhe für alle in Wien vorhandenen seismologischen Messstationen mit bekannter Instrumentenkorrektur, welche in Tabelle 3-1 aufgelistet sind. Für die Berechnung und Erstellung der Grafiken wurde „SeismoRMS“ [13] verwendet und für lokal gespeicherte Daten adaptiert.

\section{Darstellungsformen mit Beispielen der Station VIE}

Um anthropogene Quellen zu finden und analysieren können, ist die in Abbildung 4-2 gewählte Darstellung der spektralen Leistungsdichte pro Station suboptimal. Beispielhaft dafür ist Ergebnis für die Station VIE auf der Hohen Warte in Wien für 2 Wochen vor und 2 Wochen nach am 16. März in Abbildung 4-2 dargestellt. Eine Veränderung der Bodenunruhe kann hier nur schwer festgestellt werden, da die aggregierten Werte nur langfristige Muster verstärken. Der Unterschied ist nur für Perioden kleiner 1 Sekunde sichtbar. Weiters sind zeitliche Muster im Tages-, Wochen- und Jahresverlauf mit PSD-Grafiken nur schwer erkennbar. Menschliche Aktivität erfolgt aber oft in zeitlich wiederholenden Mustern, wie etwa bei Arbeitszeiten.

Deswegen werden um zeitliche Muster zu erkennen, meist andere Darstellungsformen verwendet. Eine davon sind Gitterkarten: Auf der X-Achse werden die Tage aufgetragen und auf der Y-Achse die Stunden. Pro Tag und Stunde wird dann der Wert der Amplitude angezeigt (siehe Abbildung 4-3). Im Wochenverlauf sind die Zeiten mit den „ruhigen“ Wochenenden deutlich sichtbar. Markant ist auch die Periode der stärksten Covid-19Maßnahmen von 16. März bis 31. März 2020. Weiters ist ein starkes sich wiederholendes Signal ab Anfang April um rund 4:00 Uhr früh (UTC) zu erkennen. Außerdem erkennt man an den leeren weißen Feldern Datenlücken.

Werden die Daten polar aufgetragen, wie in Abbildung 4-4, spricht man von einer Stundenkarte. Die Tage werden von innen nach außen aufgetragen. Ein Signal, welches sich zur selben Zeit wiederholt, wie das um rund 4:00 früh (UTC) wird noch deutlicher. Vortrag Reduzierte Bodenunruhe 2020 durch Corona (Apoloner)

2. Wiener Dynamik Tage 2021 
Eine Darstellungsform für wöchentlich wiederkehrende Signale und deren Veränderung mit der Zeit ist in Abbildung 4-5 zu sehen. Hier sind die Ergebnisse für 2 Wochen vor und 2 Wochen nach dem Beginn der Covid-19-Maßnahmen am 16. März zu sehen. Wochentags ist der Rückgang der Messwerte vor und nach dem 16. März sichtbar. Allgemein sind Samstag und Sonntag ruhiger als andere Wochentage. Dabei ist es zwischen 1:00 und 4:00 morgens (UTC) am ruhigsten.

Werden längere Zeiträume dargestellt ist es hilfreich wenn Wochentage, markante Daten wie Feiertage oder auch der Beginn der Covid-19-Maßnahmen eingezeichnet sind. Außerdem können zusätzliche Mittelwerte berechnet und eingezeichnet werden (Abbildung 4-6).

Die bis jetzt beschriebenen Darstellungsformen eignen sich für die Analyse einzelner Messstationen. Um einen Gesamtüberblick der Messstationen zu erhalten, ist eine weitere Vereinfachung notwendig. Dazu wird der wöchentliche Median pro Messstation berechnet. Für den Zeitraum von 15. Februar bis zu dem Lockdown am 16. März 2020 sind diese Ergebnisse in Abbildung 5-7 als prozentuelle Änderung gesammelt dargestellt.

\section{Veränderung der Bodenunruhe in Wien während der Covid-19-Maßnahmen}

Der Median der Amplitude wird als Kennwert für die Bodenunruhe verwendet und ist für alle Messstationen in Tabelle 2-1 angegeben. Die Werte variieren, bedingt durch sehr unterschiedliche Standorte (Schulen, Privatgebäude, Arbeitsgebäude) und Bauweisen (Sockel, Isolierung, Gerätetyp), zwischen 2 und 24 nm in Wien.

CONA im Conrad Observatorium wo der Standort speziell als geophysikalisches Observatorium ausgesucht und ausgebaut wurde, zeigt einen wesentlich niedrigeren Wert von $0.006 \mathrm{~nm}$.

Im nächsten Schritt wird der Median als Referenzwert von 100 \% für normale Bodenunruhe an der jeweiligen Messstation verwendet. Die prozentuelle Änderung in den zwei ersten Wochen nach dem 16. März 2020 ist in Tabelle 2-1 aufgelistet. An der Station UMWA hat sich die Bodenunruhe mit 34 \% am meisten reduziert. Andere Stationen zeigen hingegen keine signifikante Veränderung. An der Station SNWA ist die Bodenunruhe sogar gestiegen. Ein möglicher Grund dafür sind lokale Bauarbeiten.

Um die Veränderung in ganz Wien zu analysieren wurde ein Durchschnittswert aus allen Stationen pro Woche gerechnet. Dieser ist in Abbildung 5-7 aufgetragen. Daraus ergibt sich Vortrag Reduzierte Bodenunruhe 2020 durch Corona (Apoloner)

2. Wiener Dynamik Tage 2021 
eine mittlere Reduktion der Bodenunruhe von etwa $11 \%$. Zusätzlich wurde als Referenz die Station CONA am Conrad Observatorium separat hinzugefügt.

Um tektonische Phänomene, wie Erdbebenserien auszuschließen, wurde die Bodenunruhe mit dem österreichischem Erdbebenkatalog der ZAMG [14] verglichen. Hier war keine Korrelation vorhanden, deswegen wurden die Ergebnisse in dieser Studie nicht weiter berücksichtigt.

Weiters wurden die Daten mit den Mobilitätsdaten von Google verglichen. Für den Vergleich wurden folgende Kategorien ausgewählt, welche in etwa den möglichst ruhig gelegenen Standorten der Messstationen entsprechen: Parks (GFSA, GNLA, SNWA, GPRA) und Arbeitsstätten (VIE, ZAWA, WIWA, KMWA). Zusätzlich wird die Kategorie „Wohnstätten“ mit erhöhter Mobilitätsaktivität gezeigt, was sich durch vermehrtes Homeoffice und Home Schooling erklärt. Bodenunruhe und Mobilitätsdaten zeigen eine klare Korrelation zwischen den gemessenen Amplituden an den Messstationen und der Mobilitätsaktivität der Menschen. So sind beide Datenreihen in den Wochen nach dem 16. März 2020 reduziert. Die Amplituden der Messstationen reduzierten sich um bis zu $35 \%$ und sind ähnlich stark wie die Mobilitätsaktivitätsreduktion in Parks. Ab Mai 2020 sind die Werte wieder mit den Werten vor dem 16. März vergleichbar.

Auch die Messungen am Conrad Observatorium zeigten eine Reduktion der Bodenunruhe, denn durch die Einführung der Telearbeit waren auch dort weniger Mitarbeiter. Für den Standort liegen jedoch keine Google Mobilitätsdaten für einen Vergleich vor.

Gesamt zeigen die Messdaten, wie auch in der Studie [2] beschrieben, dass die seismische Bodenunruhe an den Messstationen mit menschlicher Aktivität und Mobilität korreliert. Damit könnte Mobilitätsaktivität auch aus seismischen Messungen abgeschätzt werden. Ein Vorteil bei der Nutzung seismischer Daten ist, dass deutlich weniger Probleme mit Datenschutz entstehen als bei Messungen mit öffentlichen Mikrofonen und der Auswertung von HandyDaten.

\section{Analyse unbekannter Signale}

Eine der Hauptaufgaben des Erdbebendienstes der ZAMG ist die Dokumentation und Analyse von Messsignalen. Zu einem großen Teil handelt es sich dabei um die Detektion Vortrag Reduzierte Bodenunruhe 2020 durch Corona (Apoloner)

2. Wiener Dynamik Tage 2021 
und Lokalisierung von Erdbeben und Sprengungen. Die Analyse erfolgt einerseits aufgrund der Signale in den Messdaten und anderseits durch Wahrnehmungsmeldungen aus der Bevölkerung. Erdbeben und Sprengungen können meist wegen des eindeutigen Signales und ihrer Lokalisierung klar zugeordnet werden.

Signale aus unbekannten Quellen benötigten auch einen systematischen Analyseansatz, wie wir am Beispiel von Daten der Wiener Bebenstationen der ZAMG vom 28. Juli 2020 zeigen. Zunächst wird kontrolliert ob das Signal auf mehreren Stationen aufgezeichnet wurde, entweder im interaktiven Analysesystem „Antelope“ [15] - welches der Erdbebendienst im Routinedienst verwendet - oder wie in Abbildung 6-8 gezeigt mit der frei verfügbaren Software „ObsPy“ [11], welche für die Forschung entwickelt wurde. Das besondere Signal war auf allen Stationen sichtbar und tauchte als erstes bei den Stationen GAKA und GNLA auf.

Aus der Laufzeitdifferenz und dem Stationsabstand ergibt sich eine mittlere Geschwindigkeit des Signales von $333 \mathrm{~m} / \mathrm{s}$, welche auf Schallausbreitung hindeutet. Eine Lokalisierung ist daher in einer Routineauswertung mit der Software „Antelope“ ohne zeitaufwändige Anpassung des Geschwindigkeitsmodelles nicht möglich. Auch sind kaum klare Ersteinsätze vorhanden. Wissenschaftliche Pakete wie QuakeMigrate [16] sind leichter anpassbar und benötigen keine Ersteinsätze, da sie die ganze Wellenformen stapeln. Sie sind dafür aber sehr rechenzeitintensiv.

Als nächstes wird das Signal über Amplitude und Frequenzinhalt charakterisiert. Die Amplitude wird abgelesen (siehe Tabelle 6-2), wobei die größten Werte bei GAKA mit 0,06 und GNLA mit $0,1 \mathrm{~mm} / \mathrm{s}$ auftreten. Weiters kann der Frequenzinhalt mit einem Spektrogramm untersucht werden, für das hier die interaktive Analysesoftware „Pyrocko“ [17] verwendet wurde. Für die Station GNLA, an welcher das Signal zuerst auftritt und die größte Amplitude gemessen wurde, ist das Spektrogramm in Abbildung 6-9 dargestellt. Das Signal enthält Frequenzen ab $2 \mathrm{~Hz}$ bis zu $100 \mathrm{~Hz}$, begrenzt durch die Abtastrate von $200 \mathrm{~Hz}$.

Weiters kann die Wiederholbarkeit des Signales analysiert werden. Bei der manuellen Durchsicht der Daten rund um das Signal, sind in diesem Beispiel mehrere ähnliche Signale erkennbar. Eine systematische Suche ist über eine laufende Kreuzkorrelation des Signales mit dem gesamten Datentag möglich, wie sie etwa in Pyrocko [15] implementiert ist.

Vortrag Reduzierte Bodenunruhe 2020 durch Corona (Apoloner)

2. Wiener Dynamik Tage 2021 
Für die Verifikation des Signales können ergänzende Daten verwendet werden. Je nach Art und Herkunft des Signals sind Gemeinden, Betriebe oder auch Medienmeldungen Informationsquellen. Beispiele sind hier Bauarbeiten, Felsstürze und Wetterereignisse. Ein Abgleich mit der Blitzdatenbank von Aldis [17] zeigt, dass im Rahmen eines Unwetters vom 28. Juli 2020 um 20:07:29 Uhr UTC bei 48.1694 nördliche Breite 16.4991 östlicher Länge ein Wolke-Erde-Blitz mit einer Amplitude von 196.8 kA lokalisiert wurde. Dieser befand sich in knapp 4 km Entfernung von den nächstliegenden Messstationen GAKA und GNLA. Mit der abgeschätzten Geschwindigkeit wurde das Signal als das zum Blitz zugehörige Donnern identifiziert.

Tabelle 2-1: $\quad$ Durch den Erdbebendienst der ZAMG betriebene seismische Messstationen in Wien und Rückgang der Bodenunruhe 2 Wochen nach dem Lockdown

\begin{tabular}{|c|c|c|c|c|}
\hline Messstation & $\begin{array}{r}\text { Breite } \\
{\left[{ }^{\circ}\right]}\end{array}$ & $\begin{array}{r}\text { Längengrad } \\
{\left[{ }^{\circ}\right]}\end{array}$ & $\begin{array}{r}\text { Bodenunruhe } \\
\text { [nm] }\end{array}$ & $\begin{array}{r}\text { Rückgang } \\
{[\%]}\end{array}$ \\
\hline UMWA, Uhrenmuseum (Keller) & 48,2108 & 16,3693 & 16 & 34 \\
\hline $\begin{array}{l}\text { KMWA, Kindergarten Kaisermühlen } \\
\text { (Keller) }\end{array}$ & 48,2301 & 16,4225 & 24 & 20 \\
\hline VIE,Hohe Warte (Keller), Wien & 48,2489 & 16,3567 & 10 & 12 \\
\hline $\begin{array}{l}\text { WIWA, Hauptschule Wieden } \\
\text { (Keller), }\end{array}$ & 48,1911 & 16,3679 & 24 & 14 \\
\hline $\begin{array}{l}\text { SNWA, Schloss Neuwaldegg } \\
\text { (Keller), }\end{array}$ & 48,2347 & 16,288 & 2 & -4 \\
\hline BGWA,Palais Festetics (Keller) & 48,2182 & 16,3626 & 23 & 12 \\
\hline $\begin{array}{l}\text { GAKA, Alte Kaserne (EG), } \\
\text { Groß Enzersdorf }\end{array}$ & 48,1910 & 16,5443 & 10 & 8 \\
\hline GNLA, Naturistenpark Lobau (EG) & 48,2042 & 16,4894 & 12 & 28 \\
\hline GFSA, Friedhof Süßenbrunn (EG) & 48,2765 & 16,5011 & 8 & 1 \\
\hline GPRA, Pumphaus (EG), Raasdorf & 48,2461 & 16,5603 & 8 & 20 \\
\hline ZAWA, Hohe Warte (Keller) & 48,2483 & 16,3617 & 11 & 9 \\
\hline $\begin{array}{l}\text { CONA, Conrad Observatorium } \\
\text { (Stollen), Niederösterreich }\end{array}$ & 47,9282 & 15,8618 & 0,006 & 11 \\
\hline
\end{tabular}

Vortrag Reduzierte Bodenunruhe 2020 durch Corona (Apoloner)

2. Wiener Dynamik Tage 2021 
Vortrag Reduzierte Bodenunruhe 2020 durch Corona (Apoloner)

2. Wiener Dynamik Tage 2021 
Tabelle 2-2: $\quad$ Maximale Amplitude der Signale vom 28. Juli 2020 um 20:07 Uhr UTC auf ausgewählten Messstationen der ZAMG, Distanz zu Wolke-Erde-Blitz von ALDIS [17]

\begin{tabular}{|l|r|r|}
\hline \multicolumn{1}{|r|}{ Messstation } & Amplitude & Distanz \\
\hline GNLA & $0,0100 \mathrm{~cm} / \mathrm{s}$ & $3,7 \mathrm{~km}$ \\
\hline GAKA & $0,0060 \mathrm{~cm} / \mathrm{s}$ & $4,0 \mathrm{~km}$ \\
\hline GPRA & $0,0025 \mathrm{~cm} / \mathrm{s}$ & $9,5 \mathrm{~km}$ \\
\hline GFSA & $0,0020 \mathrm{~cm} / \mathrm{s}$ & $11,7 \mathrm{~km}$ \\
\hline VIE & $0,0007 \mathrm{~cm} / \mathrm{s}$ & $13,4 \mathrm{~km}$ \\
\hline KMWA & $1,0000 \mathrm{~cm} / \mathrm{s}^{2}$ & $8,7 \mathrm{~km}$ \\
\hline WIWA & $0,5000 \mathrm{~cm} / \mathrm{s}^{2}$ & $10,0 \mathrm{~km}$ \\
\hline UMWA & $0,4000 \mathrm{~cm} / \mathrm{s}^{2}$ & $10,5 \mathrm{~km}$ \\
\hline BGWA & $0,2000 \mathrm{~cm} / \mathrm{s}^{2}$ & $11,5 \mathrm{~km}$ \\
\hline ZAWA & $0,1000 \mathrm{~cm} / \mathrm{s}^{2}$ & $13,7 \mathrm{~km}$ \\
\hline SNWA & $0,0800 \mathrm{~nm} / \mathrm{s}^{2}$ & $17,2 \mathrm{~km}$ \\
\hline
\end{tabular}

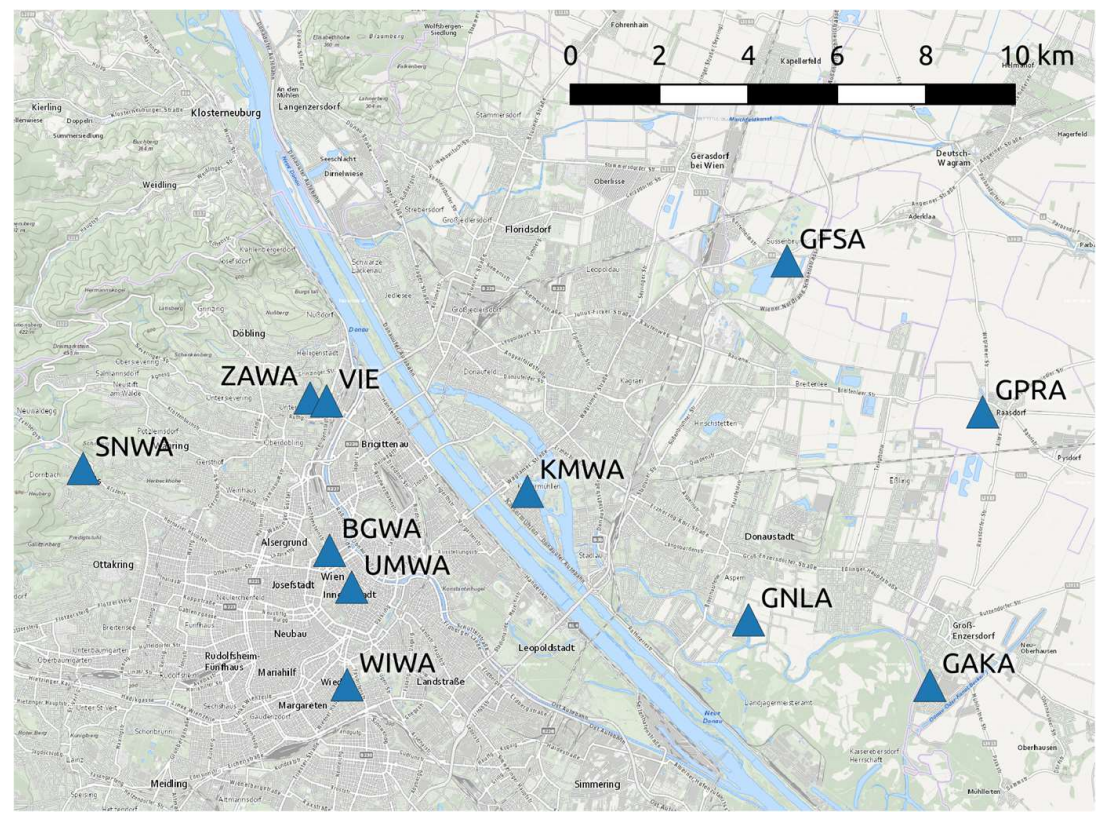

Abbildung 2-1: Karte der seismischen Messstationen der ZAMG in Wien. Erstellt mit „QGIS“ [19] und Kartenhintergrund von „basemap.at“

Vortrag Reduzierte Bodenunruhe 2020 durch Corona (Apoloner)

2. Wiener Dynamik Tage 2021 


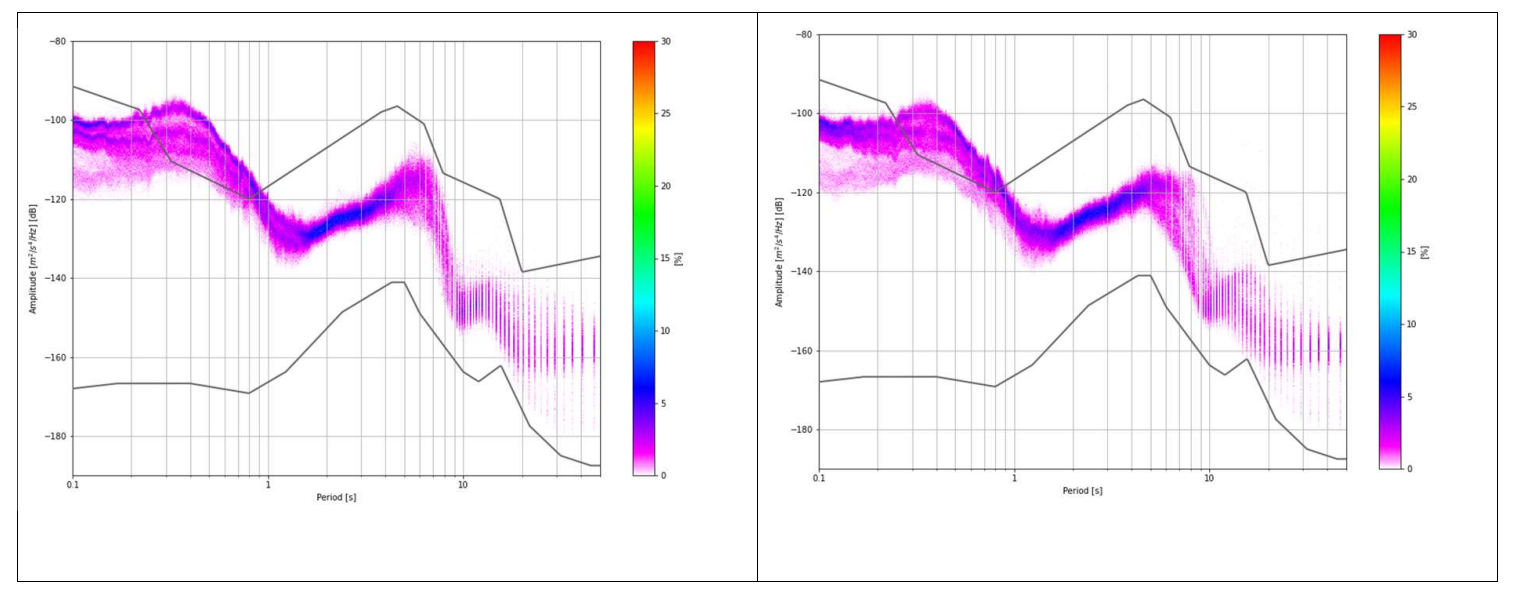

Abbildung 4-2: Spektrale Leistungsdichte A [dB] an der ZAMG Erdbebenmessstation VIE auf der Horizontalkomponente HHZ, links: 2 Wochen vor dem 16. März 2020, rechts: 2 Wochen danach. Bei den Perioden 0,1 bis 1 Sekunden ist ein kleiner Rückgang zu sehen.

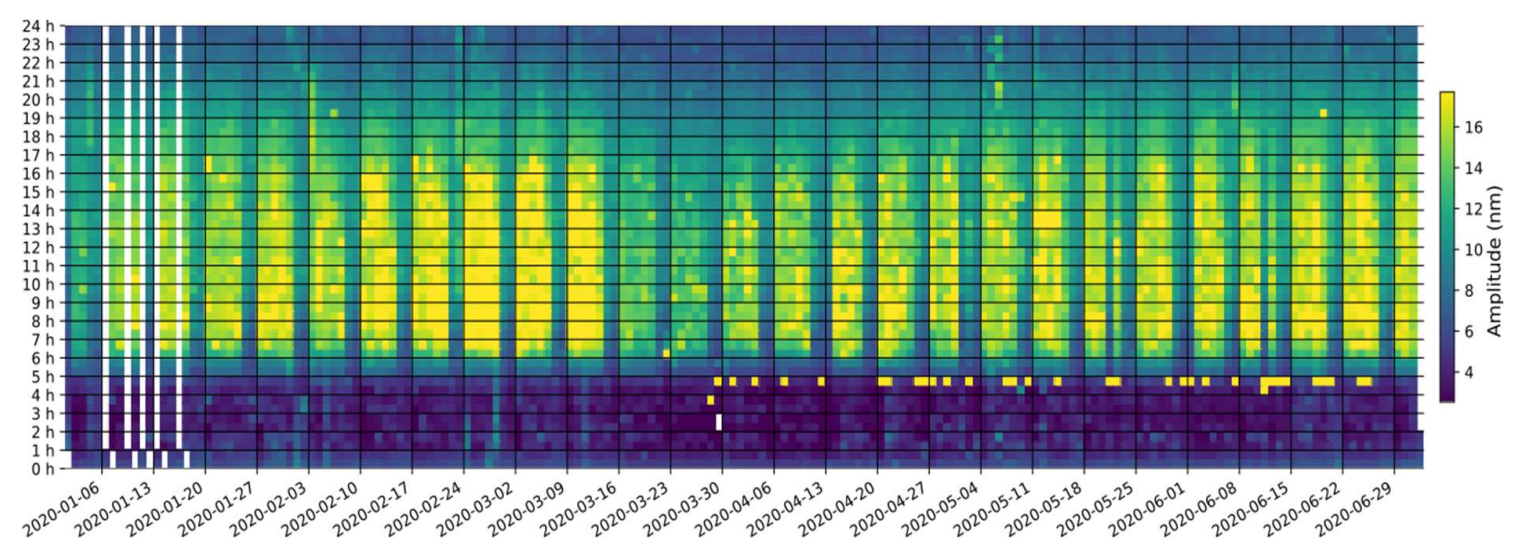

Abbildung 4-3: Gitterkarte der Amplitude der Bodenunruhe zwischen 4 und $14 \mathrm{~Hz}$ an der Station VIE. Für jede Stunde pro Tag wird im Gitter der Amplitudenwert aufgetragen. Weiße Felder zeigen Datenlücken.

Vortrag Reduzierte Bodenunruhe 2020 durch Corona (Apoloner)

2. Wiener Dynamik Tage 2021 


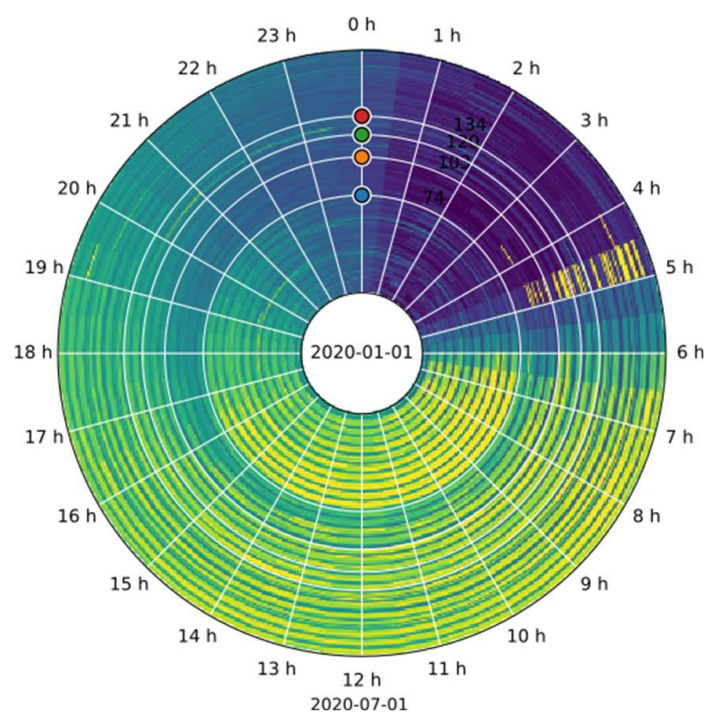

Abbildung 4-4: Stundenkarte der Amplitude der Bodenunruhe zwischen 4 und $14 \mathrm{~Hz}$ an der Station VIE. Gleiche Farbskalierung wie in Abbildung 2-4. Die Punkte um 00:00 markieren die unterschiedlichen Stufen des Corona-Lockdowns, wie detailliert in Abbildung 2 beschrieben ist. Zwischen dem blauen und orangen Punkt ist der stärkste Rückgang zu sehen.

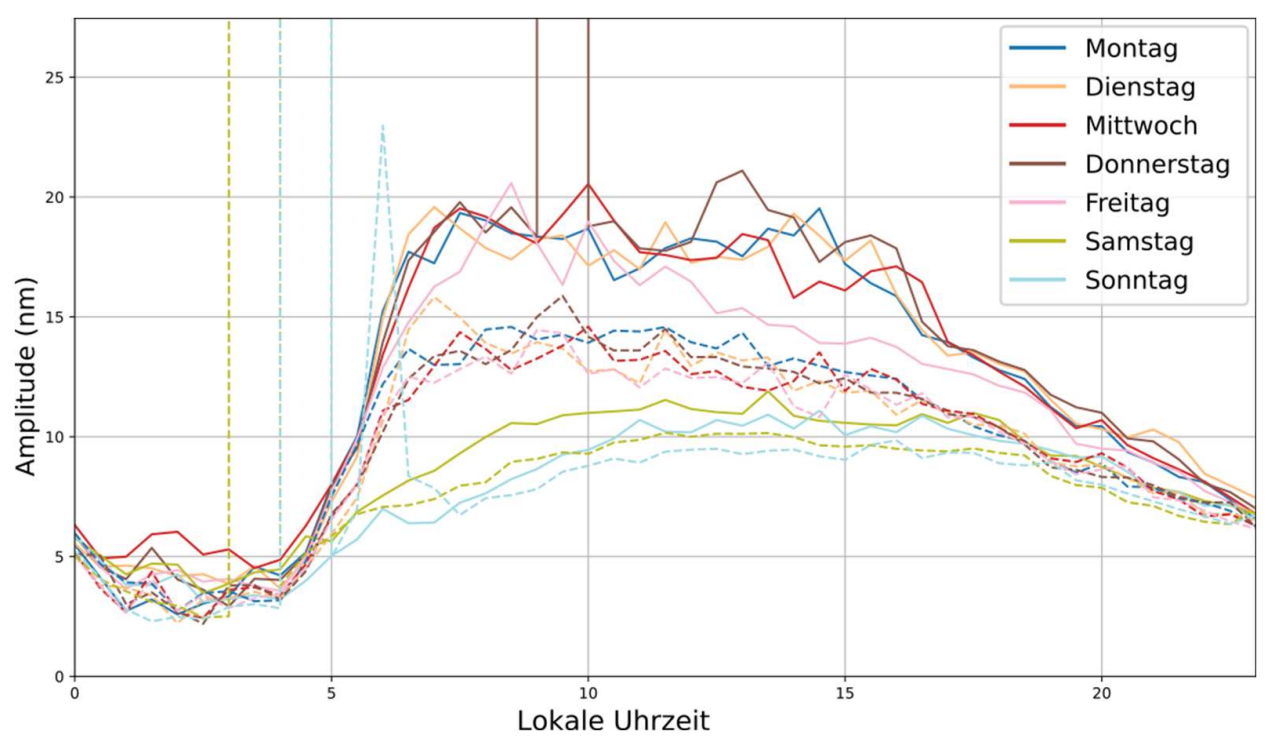

Abbildung 4-5: Tagesverlauf der Bodenunruhe auf der Station VIE im Vergleich 2 Wochen vor (durchgehend) und 2 Wochen nach (strichliert) dem Lockdown am 16. März 2020

Vortrag Reduzierte Bodenunruhe 2020 durch Corona (Apoloner)

2. Wiener Dynamik Tage 2021 


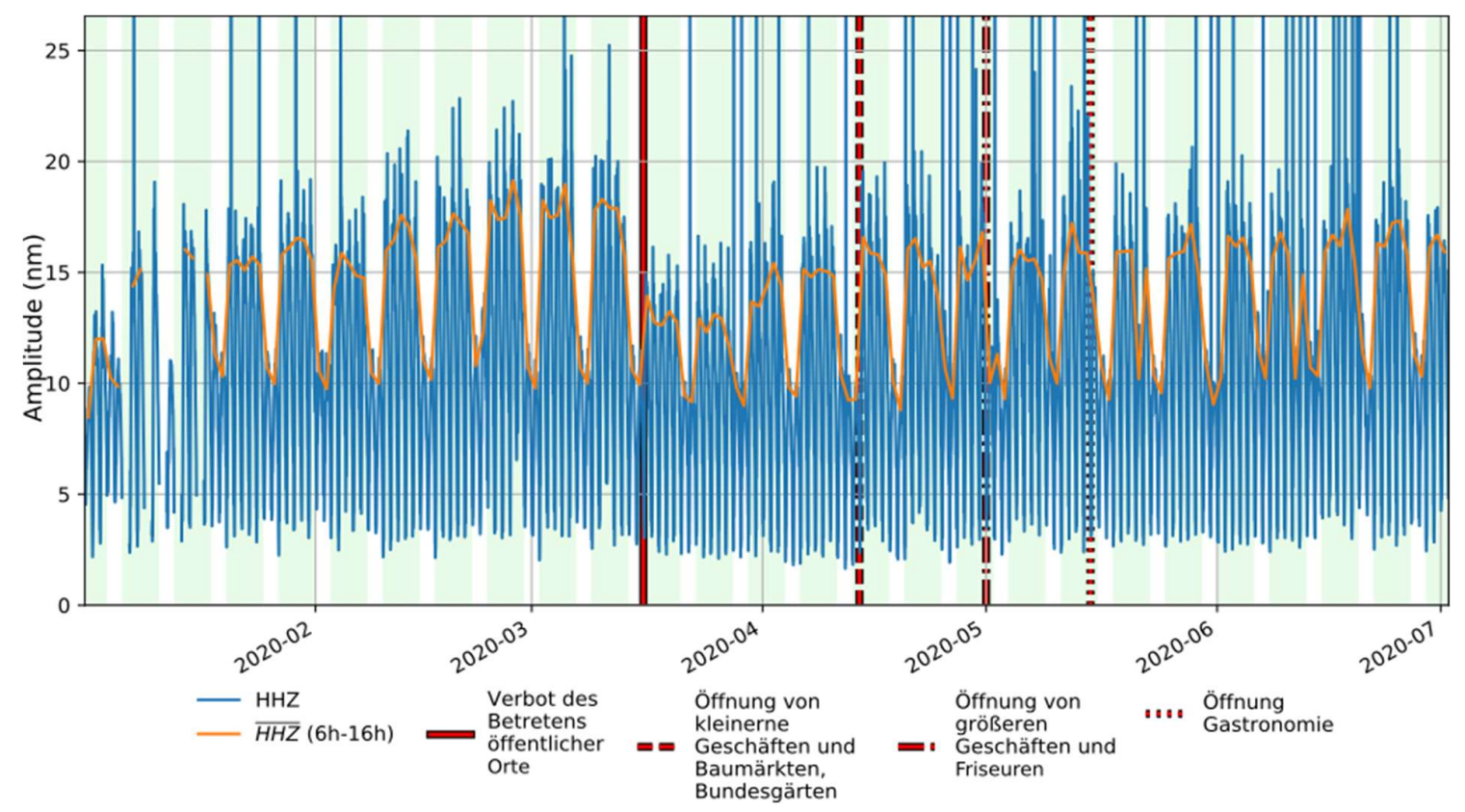

Abbildung 4-6: Veränderung der Bodenunruhe an der ZAMG BreitbandErdbebenmessstation VIE in Wien auf der Horizontalkomponente [3], Berechnung nach [2]

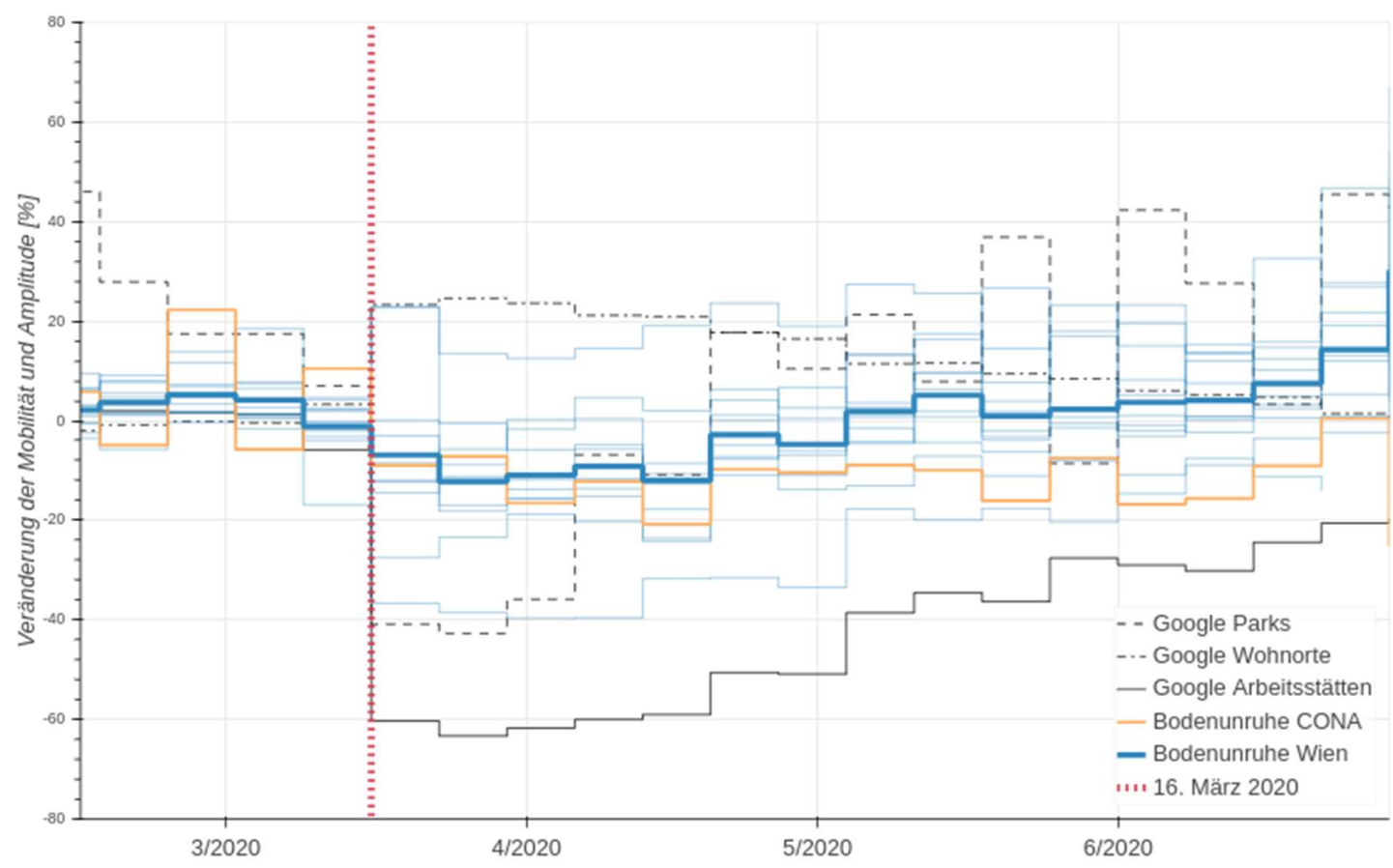

Abbildung 5-7: Entwicklung der Bodenunruhe in Wien im Vergleich mit Google Mobilitätsdaten [9]

Vortrag Reduzierte Bodenunruhe 2020 durch Corona (Apoloner)

2. Wiener Dynamik Tage 2021 


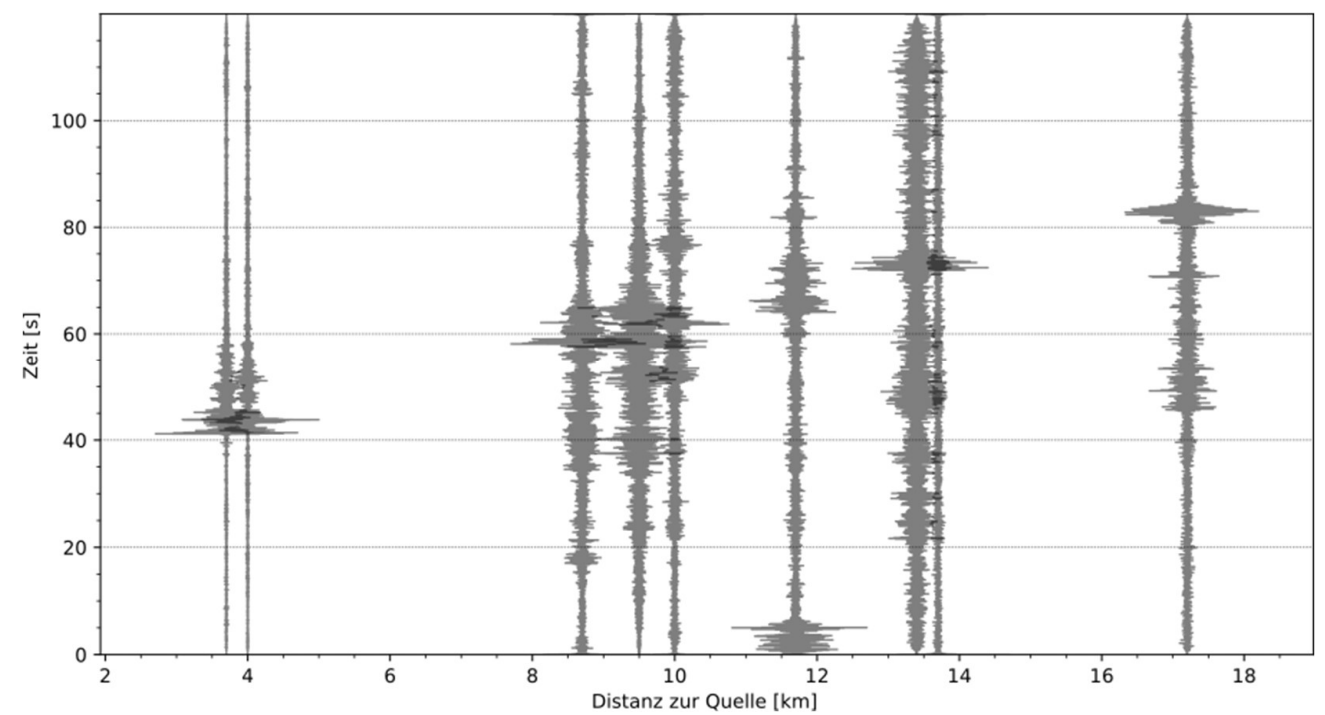

Abbildung 6-8: Aufzeichnung von Wellenformdaten an Wiener Messstationen vom 28. Juli 2020 von 1 bis $10 \mathrm{~Hz}$ gefiltert. Die Messstationen sind nach der Distanz zur Quelle sortiert. Grafik erstellt mit „ObsPy“[11]

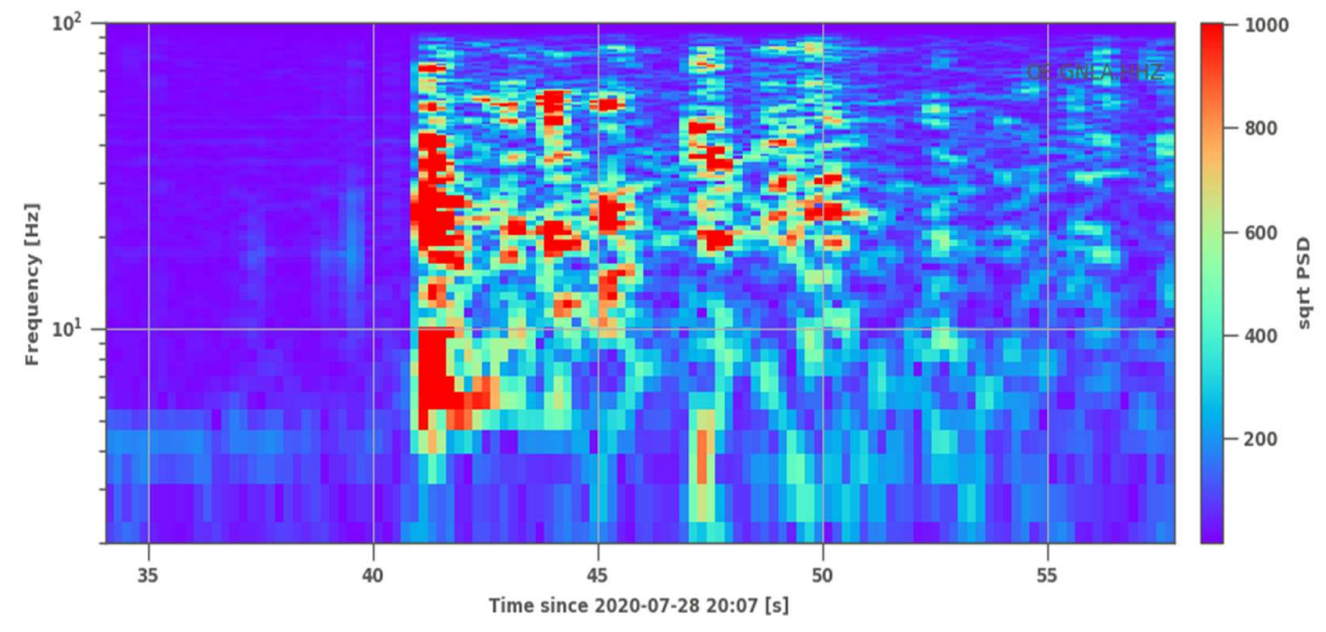

Abbildung 6-9: Spektrogramm der Station mit dem stärksten Signal GNLA. Grafik erstellt mit „Pyrocko“ [16]

\section{Zusammenfassung}

Im Rahmen dieser Studie analysieren wir die kontinuierlich aufgezeichneten Daten der seismischen Messstationen der ZAMG in und nahe bei Wien. Wir verwenden das PythonPaket SeismoRMS [13] um die mittlere Bodenunruhe an den einzelnen Messstandorten zu Vortrag Reduzierte Bodenunruhe 2020 durch Corona (Apoloner)

2. Wiener Dynamik Tage 2021 
bestimmen und zeigen anschließend unterschiedliche Darstellungsformen für seismische Bodenunruhe.

Für Wien ergibt sich gesamt ein Rückgang der Bodenunruhe von etwa $10 \%$ in den ersten 2 Wochen nach dem Inkrafttreten der Covid-19-Maßnahmen am 16. März 2020. Ab Anfang Mai kehren die Messwerte wieder zu Werten wie vor Beginn der Covid-19-Maßnahmen zurück. Derselbe Trend, allerdings stärker ausgeprägt ist auch in den Google Mobilitätsdaten [9] zu sehen.

Im Abschluss zeigen wir noch die Signalanalyse eines „unbekannten“ Signals welches abends am 28. Juli 2020 aufgezeichnet wurde. Dazu verwenden wir unterschiedliche frei verfügbare Softwarepakete [11][17]. Im Abgleich mit dem Blitzortungssystems ALDIS [18] kann das Signal eindeutig als Donner identifiziert werden.

\section{Literaturangaben:}

[1] W. Lenhardt, G. Gangl, und R. Flesch (2001). Erdbebengefährdung in Österreich. In: C. Hammerl, W. Lenhardt, R. Steinacker, P. Steinhauser (Hg.): Die Zentralanstalt für Meteorologie und Geodynamik 1851-2001. 150 Jahre Meteorologie und Geophysik in Österreich (Leykam Verlag 2001)

[2] T. Lecocq, S. P. Hicks, K. Van Noten, K. van Wijk, PI Koelemeijer, R. S. M. De Plaen, F. Massin, G. Hillers, R. E. Anthony, M.-T. Apoloner, M. ArroyoSolórzano, J. D. Assink, P. Büyükakpınar, A. Cannata, F. Cannavo, S. Carrasco, C. Caudron, E. J. Chaves, D. G. Cornwell, D. Craig, O. F. C. den Ouden, J. Diaz, S. Donner, C. P. Evangelidis, L. Evers, B. Fauville, G. A. Fernandez, D. Giannopoulos, S. J. Gibbons, T. Girona, B. Grecu, M. Grunberg, G. Hetényi, A. Horleston, A. Inza, J. C. E. Irving, M. Jamalreyhani, A. Kafka, M. R. Koymans, C. R. Labedz, E. Larose, N. J. Lindsey, M. McKinnon, T. Megies, M. S. Miller, W. Minarik, L. Moresi, V. H. Márquez-Ramírez, M. Möllhoff, I. M. Nesbitt, S. Niyogi, J. Ojeda, A. Oth, S. Proud, J. Pulli, L. Retailleau, A. E. Rintamäki , C. Satriano, M. K. Savage, S. ShaniKadmiel, R. Sleeman, E. Sokos, K. Stammler, A. E. Stott, S. Subedi, M. B. Sørensen, T. Taira, M. Tapia, F. Turhan, B. van der Pluijm, M. Vanstone, J. Vergne, T. A. T. Vuorinen, T. Warren, J. Wassermann, H. Xiao (2020). Global quieting of high-frequency seismic noise due to COVID-19 pandemic lockdown measures. Science. doi:10.1126/science.abd2438 [3] ZAMG Zentralanstalt für Meteorologie und Geodynamik (1987). Austrian Seismic Network. International Federation of Digital Seismograph Networks. doi:10.7914/SN/OE Vortrag Reduzierte Bodenunruhe 2020 durch Corona (Apoloner) 
[4] M. E. Kappus and F. L. Vernon (1991). Acoustic signature of thunder from seismic records. Journal of Geophysical Research, 96(D6), 10989. doi:10.1029/91jd00789

[5] J. Diaz, M. Schimmel, M. Ruiz und R. Carbonell (2020). Seismometers within Cities: A Tool to Connect Earth Sciences and Society. Frontiers in Earth Science.

doi:10.3389/feart.2020.00009

[6] J. Diaz (2020). Church bells and ground motions. Journal of Seismology. doi:10.1007/s10950-020-09935-2

[7] G. Duma, N. Horn und A. Vogelmann (1996). Seismisches Strong-Motion Messnetz in Wien. Endbericht im Auftrag des Bundesministeriums für Wissenschaft \& Forschung und der Stadt Wien.

[8] M.-T. Apoloner, F.-A. Rodler, Y. Jia, S. Weginger und W. Lenhardt (2019). Preliminary seismological monitoring for geothermal development in Vienna, Austria. In EGU General Assembly 2019. EGU.

[9] Google (2020). Community Mobility Reports. Online:

https://www.google.com/covid19/mobility/ (24.07.2020)

[10] D. E. McNamara und R. P. Buland (2004). Ambient Noise Levels in the Continental United States. Bulletin of the Seismological Society of America, 94 (4), 1517-1527.

[11] L. Krischer, T. Megies, R. Barsch, M. Beyreuther, T. Lecocq, C. Caudron und J. Wassermann (2015). ObsPy: a bridge for seismology into the scientific Python ecosystem. Computational Science \& Discovery, 8(1), 014003. doi: 10.1088/1749-4699/8/1/014003 [12] J. Peterson (1993). Observations and Modeling of Seismic Background Noise. U.S. Geological Survey open-file report 93-322, Albuquerque, N. M.

[13] T. Lecocq, F. Massin, C. Satriano, M. Vanstone und T. Megies (2020). SeismoRMS - A simple python/jupyter notebook package for studying seismic noise changes. Online: https://zenodo.org/record/3820046\#.XyKUHHUzZhF (11.05.2020)

[14] Austrian Earthquake Catalog (AEC) (2020). Austrian Earthquake Catalogue from 1000 to 2020 A.D., Zentralanstalt für Meteorologie und Geodynamik, Abteilung Geophysik, 1190 Wien.

[15] Antelope Environmental Monitoring Software (1996). Boulder Real Time Technologies Inc. Kinemetrics.

Vortrag Reduzierte Bodenunruhe 2020 durch Corona (Apoloner)

2. Wiener Dynamik Tage 2021 
[16] C. A. Bacon, J. D. Smith, T. Winder, T. Hudson, T. Greenfield und R. S. White (2019). QuakeMigrate: a Modular, Open-Source Python Package for Earthquake Detection and Location. In AGU Fall Meeting 2019. AGU.

[17] S. Heimann, M. Kriegerowski, M. Isken, S. Cesca, S. Daout, F. Grigoli, C. Juretzek, T. Megies, N. Nooshiri, A. Steinberg, H. Sudhaus, H. Vasyura-Bathke., T. Willey, und T. Dahm (2017) Pyrocko - An open-source seismology toolbox and library. V. 0.3. GFZ Data Services. Doi:10.5880/GFZ.2.1.2017.001

[18] ALDIS Expert Web (2020). Austrian Lightning Detection and Information System. Online: http://aldis.at (29.07.2020)

[19] QGIS Development Team, QGIS Geographic Information System, Open Source Geospatial Foundation (2020). Online: http://qgis.org (29.07.2020)

Autorenkontakt: maria-theresia.apoloner@zamg.ac.at

Vortrag Reduzierte Bodenunruhe 2020 durch Corona (Apoloner)

2. Wiener Dynamik Tage 2021 